\title{
Calidad de vida en los pacientes con DM-2: Un estudio transversal en el sureste mexicano
}

\author{
Quality of life in patients with DM-2: A cross-sectional study in the Mexican southeast \\ Crystell Guadalupe Guzmán-Priego', Guadalupe del Carmen Baeza-Flores', Raúl Guzmán-León'1, Jorge Elías Torres-López¹,2, \\ Karla Krystel Ordaz-Candelario², Juan Manuel Muñoz-Cano'
}

\section{Resumen}

La diabetes es una enfermedad crónica y compleja con demandas y desafíos que influyen en la calidad de vida relacionada a la salud. Objetivo: determinar la calidad de vida en los pacientes con diabetes mellitus tipo 2 que acuden a los servicios médicos de la UJAT durante el periodo junio- julio del 2017. Métodos: se realizó un estudio transversal en 80 sujetos con diagnóstico de diabetes mellitus tipo 2 que acuden a un primer nivel de atención en el sureste mexicano. Se diseñó un instrumento y se obtuvieron datos sociodemográficos, de calidad de vida, antropométrica, bioquímica y clínica. Las variables fueron analizadas a través de estadística descriptiva, además, se utilizó la prueba de Chi cuadrado para explorar asociación entre variables categóricas y para variables continuas la prueba de T, considerando los estadísticamente significativos los resultados de $p<0,05$. Resultados: la media de edad de los pacientes estudiados fue 57,8 años. El dominio de la calidad de vida principalmente afectado fue energía, funcionamiento sexual y movilidad, y los factores que tuvieron efecto sobre la calidad de vida fueron la escolaridad, las comorbilidades, la polifarmacia, el índice de masa corporal y la hiperglucemia. Discusión: los pacientes con diagnóstico de diabetes mellitus tipo 2 que acuden a un primer nivel institucional del sureste mexicano están principalmente afectados en 3 dominios: energía y movilidad, ansiedad y preocupación y funcionamiento sexual.

Palabras clave: diabetes mellitus tipo 2; calidad de vida; polifarmacia; hiperglucemia; ansiedad; escolaridad.

\begin{abstract}
Diabetes is a chronic and complex disease with demands and challenges that influence the quality of life - related to health. Objective: To determine the quality of life in patients with type 2 diabetes in the first level of care in Tabasco, during June- July 2017. Methods: A cross-sectional study was carried out in 102 subjects with type 2 diabetes. An instrument collected sociodemographic, quality of life, anthropometric, biochemical, and clinical data. The variables analyzed through descriptive statistics, also, the Chi-square test to explore the association between categorical variables, and for continuous variables, the T-test. Finally, considering the statistically significant results of $p<0,05$. Results: The mean age of patients included was 57,8 years old. The domain of the quality of life principally affected were energy-mobility, anxiety-concern, and sexual function, and the factors that affected the quality of life were education, comorbidities, polypharmacy, body mass index, and hyperglycemia. Discussion: Patients with type 2 diabetes in the first institutional level in the Mexican south are principally affected in three domains: energy-mobility, anxiety-concern, and sexual function.
\end{abstract}

Keywords: diabetes mellitus type 2; quality of life; polypharmacy; hyperglycemia; anxiety; educational status

Fecha de envío: 14 de agosto de 2019 - Fecha de aceptación: 11 de marzo de 2020

\section{Introducción}

La diabetes es definida por la Asociación Americana de Diabetes (ADA) como una enfermedad crónica y compleja que demanda mayor atención que solo el control glucémico (ADA, 2019d). De acuerdo a la clasificación se categoriza en 4 tipos, pero cerca del $95 \%$ está representada por la diabetes tipo 2, que se caracteriza por la pérdida progresiva de la secreción de insulina pancreática (ADA, 2019a) con desafíos y demandas a nivel físico, emocional y social (Rubin \& Peyrot, 1999).

En México en el año 2011, se registró que las muertes por diabetes fue el $11,7 \%$ en hombres y $4,6 \%$ por encima en mujeres, superando a países como Chile, Argentina y Colombia (Agudelo \& Dávila-Cervantes,

(1) Centro de Investigación, División Académica de Ciencias de la Salud, Universidad Juárez Autónoma de Tabasco, Villahermosa Tabasco, México. (2) Hospital Regional de Alta Especialidad "Dr. Juan Graham Casasús", Villahermosa Tabasco, México.

Autor de correspondencia:ba_lupitabaeza@hotmail.com 
2015). Estos datos se traducen en efecto directo sobre la economía nacional, los costos reportados por el Instituto Mexicano del Seguro Social (IMSS) durante el periodo 2008-2013 por hospitalización debida diabetes fueron de 1563 millones de dólares, del cual el 80\% fueron destinados a la atención de pacientes con diabetes mellitus tipo 2 y sus complicaciones (Salas et al., 2018). En este contexto, recientemente se informó que entre las complicaciones micro y macrovasculares que afectan a la población mexicana destacan la enfermedad de pie, la enfermedad renal crónica y la retinopatía (Ovalle et al., 2019).

La calidad de vida relacionada a la salud de los individuos y factores que aumentan o disminuyen la autopercepción de salud y las dimensiones que la integran como es el caso particular de las personas con diagnóstico de diabetes mellitus ha sido materia de muchas investigaciones (Chen, et al., 2015). Estudios previos muestran que la edad, el estado civil, el sexo, la ocupación, las complicaciones, el número de comorbilidades, la duración de la diabetes y la depresión (Debnath, et al., 2018) influyen en la calidad de vida relacionada a la salud de los individuos (Zurita et al., 2018), además que el nivel educativo alto se asocia con una mejor salud (Cáceres et al., 2018).

De acuerdo al contexto, nuestro objetivo fue determinar la calidad de vida en los pacientes con diagnóstico previo de diabetes mellitus tipo II que acuden a los servicios médicos de la UJAT durante el periodo junio- julio del 2017.

\section{Materiales y método:}

Se realizó una investigación transversal. La población de estudio estuvo constituida por 13, 664 sujetos adscritos a un primer nivel de atención privada en el sureste de México, con diagnóstico previo de diabetes mellitus tipo 2 (DM2).

Se estudió una muestra no aleatoria obtenida a través de muestreo por conveniencia de 80 sujetos que aceptaron participar a través de la firma de consentimiento bajo información durante el periodo junio-julio 2017. La investigación incluyó a usuarios mayores de 18 años, de ambos sexos, con diagnóstico previo de diabetes mellitus. No fueron incluidos pacientes que no supieran leer o escribir, con diagnóstico de diabetes tipo 1 o gestacional, mujeres embarazadas o en lactancia y se eliminaron los pacientes que dejaron instrumentos de recolección incompletos.

Se elaboró una hoja de recolección de información estructurada en las secciones sociodemográfica, de calidad de vida, antropométricos, bioquímicos y clínicos.

Con respecto a las variables sociodemográficas, se incluyó la edad, sexo, escolaridad, ocupación e ingresos mensuales. Al mismo tiempo, la calidad de vida se evaluó a través del cuestionario diabetes 39 sin modificaciones, diseñado para medir la calidad de vida en pacientes con diabetes tipo 1 y 2 (Boyer \& Jo, 1997) adaptado de la versión inglesa al español en México en el año de 2006 con alto nivel de consistencia interna y test retest. El cuestionario está constituido de 39 ítems que proporcionan información de los dominios de energía y movilidad, control de la diabetes, la ansiedad y preocupación, el impacto social, funcionamiento sexual, que se responden en una escala numérica que va del 1 (nada afectado en lo absoluto) al 7 (sumamente afectado), además, en esta misma escala se evalúan 2 ítems finales, para conocer la autopercepción de la calidad de vida y la gravedad de la diabetes que se interpretan como mínima a máxima y ninguna severidad hasta extremadamente grave respectivamente (Boyer \& Jo, 1997; López \& Rodríguez, 2006). También se interrogó acerca del tiempo de evolución de la diabetes mellitus, la presencia o ausencia de comorbilidades y la polifarmacia definida como el consumo $\geq 5$ fármacos. El peso, la talla y la tensión arterial fueron medidos por el investigador principal. Los parámetros bioquímicos: glucosa (mg/dL), colesterol total ( $\mathrm{mg} / \mathrm{dL})$, triglicéridos ( $\mathrm{mg} / \mathrm{dL}$ ) y hemoglobina glucosilada (\%) se obtuvieron del último resultado disponible en el expediente clínico con un periodo no mayor a 3 meses de determinación.

\section{Análisis de datos}

Todas las variables se evaluaron a través de estadística descriptiva. Conviene subrayar que las variables sociodemográficas se sistematizaron para su manejo, la edad se clasificó en menores y mayores de 60 años (considerando que la definición de adulto mayor por la Organización Mundial de la Salud (OMS), el nivel de escolaridad se describió como $\leq$ educación media superior (hasta secundaria o similar a 12 años de estudio) y $\geq$ educación superior (que incluyó a todos los sujetos que tenían estudios de licenciatura o posgrado concluidos), con respecto a la ocupación la categoría desempleo/empleo no formal circunscribióa los sujetos con desempleo, retiro, o empleo no formal (caracterizado por falta de seguridad social) y el resto se interpretó como empleo formal.

Para conocer la calidad de vida se sumaron los resultados de cada sección y fueron transformados en una escala de 0 a 100, donde el 0 representa el menor impacto en la calidad de vida y el 100 el máximo impacto, además, se obtuvieron las medidas de tendencia central y dispersión.

Se calculó el IMC con la fórmula peso $(\mathrm{kg})$ / talla $(\mathrm{m}) 2$ y los resultados fueron clasificados de acuerdo a lo indicado en la NOM043-SSA1-2012 como normopeso los resultados $\leq 24,9,25$ a 29 sobrepeso y obesidad $\geq 30,0$ (NOM-043-SSA2-2012, 2013).

En cuanto a los parámetros bioquímicos cada resultado se clasificó según las recomendaciones. Los resultados de glucosa se clasificaron de acuerdo a los estándares 2019 de la ADA y se definió como hipoglucemia la concentración de glucosa $\geq 54$ y $<70 \mathrm{mg} / \mathrm{dL}$, normoglucemia entre 75 y $130 \mathrm{mg} / \mathrm{dL}$ e hiperglucemia $\geq 131 \mathrm{mg} /$ $\mathrm{dL}$, en relación con los resultados de la prueba $\mathrm{A} 1 \mathrm{C}$ se interpretó 
como normal $<7 \%$ y anormal $>7 \%$ (ADA, 2019b). Los resultados de la presión arterial fueron definidos como hipertensión la presión arterial $\geq 140 / 90 \mathrm{~mm} / \mathrm{Hg}$ (ADA, 2019c).

Los niveles de lípidos fueron clasificados de NOM-037-SSA2-2012 en recomendable, limítrofe y alto riesgo (Tabla 1) (NOM-037SSA2-2012, 2012).

Tabla 1: Clasificación de lípidos.

\begin{tabular}{l|l|l|l|l}
\hline & Recomendable & Limítrofe & $\begin{array}{l}\text { Alto } \\
\text { riesgo }\end{array}$ & $\begin{array}{l}\text { Muy Alto } \\
\text { Riesgo }\end{array}$ \\
\hline $\begin{array}{l}\text { Colesterol } \\
\text { total }\end{array}$ & $<200$ & $200-239$ & $\geq 240$ & - \\
\hline Triglicéridos & $<150$ & $150-200$ & $>200$ & $>1000$ \\
\hline
\end{tabular}

Para el análisis bivariado se utilizó la prueba de Chi cuadrado para explorar asociación entre variables categóricas y para variables continuas la prueba de T, considerando los estadísticamente significativos los resultados de $p<0,05$.

\section{Resultados}

\section{Características sociodemográficas}

Se estudió un total de 80 derechohabientes adscritos a los servicios médicos de la Universidad Juárez Autónoma de Tabasco, de acuerdo a la exploración de las características sociodemográficas se encontró una media de edad de 57,8 8 11,9 años con un mínimo de 26 y máximo de 90 años, la distribución por sexo reveló que el 56,3\% $(n=45)$ son mujeres, además, el 57,5\% $(n=46)$ tienen como nivel de escolaridad licenciatura o posgrado, sin embargo, más de la mitad de la población encuestada está desempleada o tiene un empleo no formal, lo que deriva en que el $55,1 \%(n=44)$ tenga un ingreso mensual menor a los 8000 pesos mexicanos ( 421 dólares) (Tabla 2).

Tabla 2: Características sociodemográficas de los derechohabientes de un primer nivel en el sureste mexicano.

\begin{tabular}{r|l} 
Edad & $57,8 \pm 11,9$ \\
\hline Grupo de edad & \\
\hline$<60$ años & $60 \%(\mathrm{n}=48)$ \\
\hline$>60$ años & $40 \%(\mathrm{n}=32)$ \\
\hline Sexo & \\
\hline Hombres & $43,8 \%(\mathrm{n}=35)$ \\
\hline Mujeres & $56,3 \%(\mathrm{n}=45)$ \\
\hline Nivel de escolaridad & \\
\hline Educación media superior & $42,5 \%(\mathrm{n}=34)$ \\
\hline Educación superior & $57,5 \%(\mathrm{n}=46)$ \\
\hline Ocupación & \\
\hline Desempleo/Empleo no formal & $53,8 \%(\mathrm{n}=43)$ \\
\hline Empleo formal & $46,3 \%(\mathrm{n}=37)$ \\
\hline Ingresos mensuales (pesos mexicanos) & \\
\hline$<8000$ & $55,1 \%(\mathrm{n}=44)$ \\
\hline$\geq 8001$ & $45,0 \%(\mathrm{n}=36)$ \\
\hline &
\end{tabular}

\section{Características clínicas, antropométricas y bioquímicas}

La tabla 3 destaca que el tiempo de evolución de la diabetes fue de 10,3 $\pm 7,3$ años, el $60 \%(n=48)$ tienen menos de 10 años de evolución, el 81,3\% ( $n=65)$ tiene comorbilidades asociadas, y cerca del $100 \%$ cursa con polifarmacia. Con respecto a las variables antropométricas, cabe resaltar que la media de IMC clasifica al grupo dentro de la categoría de obesidad, sin embargo, 86,3\% cursan con sobrepeso u obesidad de acuerdo a la clasificación. El promedio de glucosa periférica fue $179 \pm 90,7 \mathrm{mg} / \mathrm{dL}$, más de la mitad cursan con hiperglucemia, de colesterol total fue de $197,7 \pm 54,9 \mathrm{mg} / \mathrm{dL}$ sólo el 23,8\% ( $\mathrm{n=19)}$ tiene resultados de alto riesgo cardiovascular, $229,2 \pm 180,5$ fue la media de triglicéridos y por último el resultado de $\mathrm{HbA} 1 \mathrm{C}$ muestra que más de la mitad del grupo no tienen adecuado control de la enfermedad. La tensión arterial sistólica tuvo una media de $123 \pm 12.8 \mathrm{mmHg}$ y la media de tensión arterial diastólica fue 79,3 $\pm 8.8 \mathrm{mmHg}$.

Tabla 3: Características clínicas, antropométricas y bioquímicas.

\begin{tabular}{|c|c|}
\hline Tiempo de evolución de DM (años) & $10,3 \pm 7.3$ \\
\hline$<10$ años & $60 \%(n=48)$ \\
\hline$>10$ años & $40,0 \%(n=32)$ \\
\hline \multicolumn{2}{|l|}{ Comorbilidades } \\
\hline $\mathrm{Si}$ & $81,3 \%(n=65)$ \\
\hline No & $18,8 \%(n=15)$ \\
\hline \multicolumn{2}{|l|}{ Polifarmacia } \\
\hline $\mathrm{Si}$ & $93,8 \%(n=75)$ \\
\hline No & $6,3 \%(n=5)$ \\
\hline Peso $(\mathrm{Kg})$ & $79,4 \pm 20,1$ \\
\hline Talla (m) & $1,6 \pm 0,09$ \\
\hline IMC & $30,7 \pm 6,3$ \\
\hline Normopeso & $13,8 \%(n=11)$ \\
\hline Sobrepeso & $32,5 \%(\mathrm{n}=26)$ \\
\hline Obesidad & $53,8 \%(n=43)$ \\
\hline Glucosa (mg/dL) & $179,9 \pm 90.7$ \\
\hline Hipoglucemia & $1,3 \%(n=1)$ \\
\hline Normoglucemia & $38,8 \%(n=31)$ \\
\hline Hiperglucemia & $60,0 \%(\mathrm{n}=48)$ \\
\hline Colesterol total (mg/dL) & $197,7 \pm 54,9$ \\
\hline Recomendable & $51,3 \%(n=41)$ \\
\hline Limítrofe & $25,0 \%(n=20)$ \\
\hline Alto riesgo & $23,8 \%(n=19)$ \\
\hline Triglicéridos (mg/dL) & $229,2 \pm 180,5$ \\
\hline Recomendable & $36,3 \%(n=29)$ \\
\hline Limítrofe & $22,5 \%(n=18)$ \\
\hline Alto riesgo & $40,0 \%(n=32)$ \\
\hline Muy alto riesgo & $1,3 \%(n=1)$ \\
\hline HbA1C (\%) & $8,2 \pm 2,6$ \\
\hline$<7 \%$ & $48,8 \%(n=39)$ \\
\hline$>7 \%$ & $51,3 \%(n=41)$ \\
\hline \multicolumn{2}{|l|}{ Hipertensión } \\
\hline No & $88.8 \%(\mathrm{n}=71)$ \\
\hline $\mathrm{Si}$ & $11.3 \%(n=9)$ \\
\hline
\end{tabular}




\section{Calidad de vida}

En la tabla 4 se expresan los promedios por cada dominio de calidad vida de los usuarios de los servicios médicos y se observa que el dominio con mayor puntuación fue energía y movilidad y el de menor promedio fue impacto social, sin embargo, la media

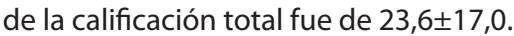

Tabla 4: Dominios de la calidad de vida.

\begin{tabular}{l|l}
\hline Dominios de la Calidad de Vida & $\square \pm D E$ \\
\hline Energía y movilidad & $28,6 \pm 20,4$ \\
\hline Control de la diabetes & $21,5 \pm 17,9$ \\
\hline Ansiedad y preocupación & $24,1 \pm 21,9$ \\
\hline Impacto social & $13,7 \pm 18,3$ \\
\hline Funcionamiento sexual & $22,9 \pm 30,1$ \\
\hline Calificación total & $23,6 \pm 17,0$ \\
\hline Calidad de vida & $64,5 \pm 25,2$ \\
\hline Severidad de la diabetes & $39,7 \pm 27,7$ \\
\hline M:Media \pm Desvión
\end{tabular}

$\overline{\mathrm{X}}:$ Media, \pm : Desviación estándar

Los pacientes con sobrepeso-obesidad tienen mayor impacto en la calidad de vida $p<0,0117$ (Normopeso 46,9 \pm 7.3 , sobrepeso-obesidad $67,3 \pm 2,9)$. El tiempo de evolución afecta el dominio funcionamiento sexual de forma significativa $p<0,0444$ ( $<10$ años de evolución

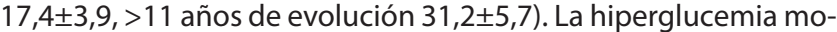
dificó la percepción de la severidad de la diabetes $p<0,0161$ (Sin

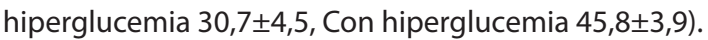

\section{Discusión:}

Nuestros resultados muestran que en pacientes con diagnóstico previo de diabetes mellitus tipo 2, en la sexta década de la vida, con predominio del sexo femenino, la calidad de vida en se ve afectada por el sobrepeso y la obesidad, por el tiempo de evolución de la DM2 y por la hiperglucemia.

También se encontró que la mayoría tiene un nivel de escolaridad alto reportado como licenciatura o posgrado, esta situación se explica ya que es una atención particular para trabajadores vigentes o retirados dedicados a la educación superior en la región. En relación con las características clínicas de la diabetes destaca que el promedio de evolución de la diabetes fue de 10,3 años, además de, una prevalencia acumulada de sobrepeso y obesidad de $86,3 \%$. Mientras tanto, los dominios de la calidad de vida que muestran mayor impacto fueron energía-movilidad, ansiedad-preocupación y funcionamiento sexual.
Conviene subrayar que el tiempo es un elemento clave en el desarrollo y precisión de los procesos fisiológicos del organismo (Salechet al., 2012), en relación con lo anterior otros autores han descrito que las enfermedades crónicas están asociadas al proceso de envejecimiento (Llibre Guerra, Guerra Hernández, \& Perera Miniet, 2008) ocasionan que la autopercepción del individuo con respecto a su entorno se modifique tal como se describió en un grupo de adultos mayores en Cuba (Gonzalez, 2019) sin embargo, nuestros datos no muestran una relación estadísticamente significativa entre la edad y los dominios que integran la calidad de vida.

Nuestros datos muestran que las mujeres son las que tienen mayor impacto en la calidad de vida, por lo que es necesario recalcar que se ha establecido que el género influye en factores biológicos, psicológicos y sociales por lo que es necesario recalcar que las mujeres frecuentemente gozan una calidad de vida relacionada a la salud disminuida (Miranda Félix et al., 2019), similar a nuestros datos en Brasil se reportó que el sexo femenino es un factor de riesgo para la peor calidad de vida (Corrêa et al., 2017). Contrario a lo anterior, en el occidente de México se describió que los hombres están negativamente afectados en el dominio funcionamiento sexual y en el dominio energía y movilidad (Estrada et al., 2012). La formación técnica y profesional de los hombres y las mujeres según la Organización de las Naciones Unidas para la Educación, la Ciencia y la Cultura (UNESCO por sus siglas en inglés) (UNESCO, 2019) permite asegurar condiciones de igualdad a través desarrollo intelectual de la población, además, se ha expuesto la relación directamente proporcional entre la escolaridad y la calidad de vida (Cáceres et al., 2018) a diferencia de nuestros datos, donde no se encontró ningún tipo de relación con los dominios.

Las comorbilidades interpretadas como entidades patológicas agregadas a la diabetes están reportadas con una prevalencia del $80 \%$ hasta cerca del $100 \%$ de la población, con variaciones principalmente identificadas geográficamente (Adriaanse et al., 2016; García et al., 2017). En este contexto se ha reportado que la mala calidad de vida es un factor predictivo para patologías como sarcopenia (Casals et al., 2017), sin embargo, no se encontró relación de la polifarmacia y comorbilidades con la calidad de vida de los pacientes con DM2 de un primer nivel de atención en Tabasco.

Nuestros resultados muestran una prevalencia de sobrepeso y obesidad fue de casi el $90 \%$ de la población, $16 \%$ por encima de los últimos reportes nacionales, exponiendo de esta manera la magnitud del problema de salud pública en nuestro estado y frecuentemente asociado a la falta de actividad física (Uribe-Carvajal et al., 2018). Los informes sobre la calidad de vida de los pacientes diabéticos con obesidad son homogéneos dentro y fuera de nuestro país, en Brasil se identificó que los pacientes diabéticos que no realizan 
ninguna actividad física tienen peor calidad de vida (Correa et al., 2017) y en España se reporta el impacto negativo de la obesidad sobre la calidad de vida a través del tiempo (Barcones et al., 2018).

De acuerdo a los protocolos para el tratamiento y objetivos terapéuticos de los pacientes con DM2, la adherencia terapéutica, los cambios en el estilo de vida, el control de los parámetros bioquímicos y $\mathrm{HbA} 1 \mathrm{C}$ se interpreta como un tratamiento exitoso. En un estudio actual realizado en los tres niveles de atención de salud en Brasil solo encontró correlación con el resultado de $\mathrm{HbA} 1 \mathrm{C}$, además, destaca que la ansiedad y la preocupación fue el dominio afectado principalmente en el primer nivel, mientras que energía y movilidad está afectado en el tercer nivel de atención, de esta forma se asume, que el deterioro de la calidad de vida es progresivo de acuerdo al nivel de atención (Tonetto et al., 2019). En cuanto a la percepción de la severidad de la diabetes se encontró que se ve alterada por la hiperglucemia.

La ansiedad y la preocupación se interpreta como parte del bienestar psicológico y emocional de los individuos, sin embargo, ha sido labor de otros autores determinar la relación entre la calidad de vida y la depresión de adultos con DM2 (Juárez et al., 2018) además, de la influencia que tiene la función cognitiva y el estado psicoemocional en calidad de vida de los pacientes con diabetes principalmente en la adherencia al tratamiento farmacológico (Babenko, et al., 2019).

En México como en otros países se han dado a la tarea de investigar acerca de las estrategias que se pueden emplear para incrementar la calidad de vida de los individuos con enfermedades crónicas como es este caso, con la finalidad de disminuir sus efectos a nivel familiar, social y poblacional, un ejemplo de esto, es la insulinización temprana o empleo de la insulina bifásica para obtener beneficios significativos sobre la movilidad, dolor, malestar, ansiedad y depresión como fue descrito en China (Yang et al., 2014), es por ello que el presente trabajo de investigación es un aporte científico de lo que sucede en el sureste mexicano y de la importancia que tiene el trabajo multidisciplinario para incrementar la calidad de vida de los pacientes con enfermedades crónicas.

\section{Conclusión}

En este estudio encontramos que los pacientes con diagnóstico de DM2 que acuden a un primer nivel institucional del sureste mexicano muestran puntuaciones más altas en 3 de los 5 dominios que integran la calidad de vida: energía - movilidad, ansiedad - preocupación y funcionamiento sexual. Además, de manera particular, identificamos como factores que afectan alguno de los dominios de la calidad de vida el sexo femenino, el sobrepeso y obesidad, el tiempo de evolución mayor a 10 años y la hiperglucemia. Por último, se destaca la importancia que tiene el estilo de vida, y la adherencia al tratamiento para incrementar la autopercepción de la calidad de vida.

\section{Fuentes de financiamiento}

Ninguno

\section{Contribuciones y conflictos declarados por los autores}

Los autores declaran que no existe ningún tipo de conflicto.

Todos los autores participaron en el diseño, análisis de datos y redacción de la versión final del artículo.

\section{Referencias:}

ADA. (2019a). 2. Classification and Diagnosis of Diabetes: Standards of Medical Care in Diabetes_2019. Diabetes Care 42(Supplement 1), 13-28.

ADA. (2019b). 6. Glycemic Targets: Standards of Medical Care in Diabetes-2019. Diabetes Care, 42(Supplement 1), 61-70.

ADA. (2019c). 10. Cardiovascular Disease and Risk Management: Standards of Medical Care in Diabetes-2019. Diabetes Care 42(Supplement 1), 103-123.

ADA. (2019d). Introduction:Standards of Medical Care in Diabetes-2019. Diabetes Care 42(Supplement 1), 1-2.

Adriaanse MC, Drewes HW, van der Heide I, Struijs JN \& Baan CA. (2016). The impact of comorbid chronic conditions on quality of life in type 2 diabetes patients. Quality of life research: an international journal of quality of life aspects of treatment, care and rehabilitation 25, 175-182.

Agudelo-Botero M \& Dávila-Cervantes CA. (2015). Carga de la mortalidad por diabetes mellitus en América Latina 2000-2011: los casos de Argentina, Chile, Colombia y México. Gaceta sanitaria 29, 172-177.

Babenko AY, Mosikian AA, Lebedev DL, Khrabrova EA \& Shlyakhto EV. (2019). Mental state, psychoemotional status, quality of life and treatment compliance in patients with Type 2 diabetes mellitus. Journal of Comparative Effectiveness Research 8, 113-120.

Barcones-Molero MF, Sánchez-Villegas A, Martínez-González MA, Bes-Rastrollo M, Martínez-Urbistondo M, Santabárbara J \& Martínez JA. (2018). Influencia de la obesidad y la ganancia de peso sobre la calidad de vida según el SF-36 en individuos de la cohorte dinámica Seguimiento Universidad de Navarra. Revista Clínica Española 218, 408-416. 
Boyer JG \& Jo Anne LE. (1997). The Development of an Instrument for Assessing the Quality of Life of People with Diabetes: Diabetes-39. Medical Care 35, 440-453.

Cáceres-Manrique FdM, Parra-Prada LM \& Pico-Espinosa OJ. (2018). Calidad de vida relacionada con la salud en población general de Bucaramanga, Colombia. Revista de Salud Pública 20, 147-154.

Casals C, Suárez-Cadenas E, Estébanez Carvajal FM, Aguilar Trujillo MP, Jiménez Arcos MM \& Vázquez Sánchez MÁ. (2017). Relación entre calidad de vida, actividad física, alimentación y control glucémico con la sarcopenia de adultos mayores con diabetes mellitus tipo 2. Nutrición Hospitalaria 34, 1198-1204.

Chen G, lezzi A, McKie J, Khan MA \& Richardson J. (2015). Diabetes and quality of life: Comparing results from utility instruments and Diabetes-39. Diabetes Research and Clinical Practice 109, 326-333. Corrêa K, Gouvêa GR, Silva MAVd, Possobon RdF, Barbosa LFdLN, Pereira AC, Cortellazzi KL (2017). Qualidade de vida e características dos pacientes diabéticos. Ciência \& Saúde Coletiva 22, 921-930.

Debnath S, O'Connor J, Hura C, Kasinath B \& Lorenzo C. (2018), Quality of Life and Depression Among Mexican Americans on Hemodialysis: A Preliminary Report. Therapeutic Apheresis and Dialysis 22, 166-170.

Estrada JGS, Strauss AMG, Beltrán CA, Baltazar RG \& Moreno MP. (2012). La calidad de vida en adultos con diabetes mellitus tipo 2 en centros de salud de Guadalajara, Jalisco (México). Salud Uninorte 28, 264-275.

Felipe Salech M, Rafael Jara L \& Luis Michea A. (2012). Cambios fisiológicos asociados al envejecimiento. Revista Médica Clínica Las Condes 23, 19-29.

García-Soidán F, Villoro R, Merino M., Hidalgo-Vega Á, Hernando-Martín T \& González-Martín-Moro B. (2017). Estado de salud, calidad de vida y utilización de recursos sanitarios de los pacientes con diabetes mellitus en España. SEMERGEN-Medicina de Familia 43, 416-424.

Gonzalez GE. (2019). Percepción de calidad de vida de trabajadores de una institución universitaria colombiana. Revista Cubana de Enfermería 35.

Juárez-Rojop I E, Fortuny-Falconi CM, González-Castro TB, ToviIla-Zárate CA, Villar-Soto M, Sanchez ER, Rodríguez-Pérez JM. (2018). Association between reduced quality of life and depression in patients with type 2 diabetes mellitus: a cohort study in a Mexican population. Neuropsychiatric disease and treatment 14, 2511-2518.
Llibre Guerra JC, Guerra Hernández MA \& Perera Miniet E. (2008).

Comportamiento de las enfermedades crónicas no transmisibles en adultos mayores. Revista Cubana de Medicina General Integral

24, 0-0.

López-Carmona JM \& Rodríguez - Moctezuma R. (2006). Adaptación y validación del instrumento de calidad de vida Diabetes 39 en pacientes mexicanos con diabetes mellitus tipo 2. Salud pública de México 48, 200-211.

Miranda Félix PE, Buichia Sombra FG, Miranda Cota GA, García Sarmiento JL \& Ortiz Félix RE. (2019). Diabetes y Género desde una Perspectiva Sociocultural $=$ Diabetes and Gender from a Sociocultural Perspective. 2019, 13.

NOM-037-SSA2-2012. (2012). I. Norma Oficial Mexicana NOM037-SSA2-2012, para la prevención, tratamiento y control de las dislipidemias. Diario Oficial de la Federación.

NOM-043-SSA2-2012, N. o. m. (2013). Servicios básicos de salud. Promoción y educación para la salud en materia alimentaria. Diario Oficial de la Federación.

Ovalle-Luna, O. D., Jiménez-Martínez, I. A., Rascón-Pacheco, R. A., Gómez-Díaz, R. A., Valdez-González, A. L., Gamiochipi-Cano, M., Méndez-Padrón, A. (2019). Prevalencia de complicaciones de la diabetes y comorbilidades asociadas en medicina familiar del Instituto Mexicano del Seguro Social. Gaceta medica de Mexico, 155(1), 30-38

Rubin RR. \& Peyrot M. (1999). Quality of life and diabetes. Diabetes/ Metabolism Research and Reviews 15, 205-218.

Salas-Zapata L, Palacio-Mejía LS, Aracena-Genao B, Hernández-Ávila JE \& Nieto-López ES. (2018). Costos directos de las hospitalizaciones por diabetes mellitus en el Instituto Mexicano del Seguro Social. Gaceta sanitaria 32, 209-215.

Tonetto, I. F. d. A., Baptista, M. H. B., Gomides, D. d. S., \& Pace, A. E. (2019). Quality of life of people with diabetes mellitus. Revista da Escola de Enfermagem da USP 53.

UNESCO. (2019). Educación superior. Accedida en https://es.unesco. org/themes/educacion-superio el 04 de abril de 2019.

Uribe-Carvajal R, Jiménez-Aguilar A, Morales-Ruan MdC, Salazar-Coronel AA \& Shamah-Levy T. (2018). Percepción del peso corporal y de la probabilidad de desarrollar obesidad en adultos mexicanos. salud pública de méxico 60, 254-262. 


\section{Guzmán et al.}

Yang W, Zhuang, X, Li Y, Wang Q, Bian R, Shen, et al. (2014). Improvements in quality of life associated with biphasic insulin aspart 30 in type 2 diabetes patients in China: results from the A1 chieve ${ }^{\circledR}$ observational study. Health and quality of life outcomes 12, 137-137.
Zurita-Cruz JN, Manuel-Apolinar L, Arellano-Flores ML, Gutierrez-Gonzalez A, Najera-Ahumada AG \& Cisneros-González N. (2018). Health and quality of life outcomes impairment of quality of life in type 2 diabetes mellitus: a cross-sectional study. Health and quality of life outcomes 16, 94-94. 